\title{
AUTORRELATO DE QUEDAS E FATORES ASSOCIADOS EM IDOSOS ASSISTIDOS NA ATENÇÃO PRIMÁRIA NO MUNICÍPIO DE CAMPINAS, SP
}

\author{
SELF-REPORT OF FALLS AND ASSOCIATED FACTORS IN THE ELDERLY ASSISTED IN PRIMARY \\ HEALTH CENTERS IN THE MUNICIPALITY OF CAMPINAS, SP
}

\author{
Andrea Moscardini da Costa ${ }^{a^{*}}$, Claudinir Leonel Aere ${ }^{b^{*}}$, Maria Elena Guariento ${ }^{c^{*}}$ \\ adea_moscardini@yahoo.com.br, bleonelaere@hotmail.com, cmeguar@fcm.unicamp.br \\ *Universidade de Campinas - Campinas (SP), Brasil
}

Data de entrada do artigo: 02/01/2014

Data de aceite do artigo: 19/03/2014

\section{RESUMO}

Objetivo: Considerando a relevância do evento quedas na população idosa, buscou-se avaliar o perfil de uma amostra de idosos assistidos em dois centros de saúde do município de Campinas (SP), a partir do autorrelato de quedas em relaçấo a gênero, idade, escolaridade e grau de dependência. Métodos: Desenvolveu-se um estudo transversal, com 70 idosos (52 mulheres) seguidos nessas unidades de saúde. Foram avaliados, além dos fatores sociodemográficos, os dados referentes ao relato de quedas e o grau de independência funcional avaliado pela Medida de Independência Funcional (MIF). A análise estatística foi feita por testes qui-quadrado ou exato de Fisher e de Mann Whitney, além do coeficiente de correlaçáo de Spearman, todos com nível de significância de 5\%. Resultados: Verificou-se que 58,57\% dos idosos relataram queda no ano anterior à avaliaçấo, dos quais $37,14 \%$ tiveram ao menos uma queda e $82 \%$ eram mulheres. O relato de quedas associou-se com déficit no domínio "mobilidade" da MIF ( $p=0,0302)$. Conclusão: Confirma-se, assim, a relevância de buscar ativamente a presença de déficit de mobilidade em idosos, particularmente nos serviços de Atençấo Primária.

Palavras-chave: Idoso; acidentes por quedas; mobilidade.

\section{ABSTRACT}

Objective: Considering the importance of the event of falls in the elderly population, we sought to evaluate the profile of a sample of elderly assisted in two health centers in Campinas, SP, from selfreported falls in relation to gender, age, education and level of dependency. Method: It was developed a cross-sectional study involving 70 older adults $(52$ women) followed by these health units. In addition to socio-demographic factors, were evaluated the data related to history of falls and the degree of functional independence measured by the Functional Independence Measure (FIM). The statistical analysis was performed using the chi-square or the exact test and of Fisher and Mann Whitney, besides the Spearman correlation coefficient, all of them with significance level of $5 \%$. Results: It was found that $58.57 \%$ of seniors reported a fall in year prior to the review, of which $37.14 \%$ had at least fallen once and $82 \%$ were women. The self-reported falls were associated with deficit in the 'mobility' domain of FIM ( $p=0.0302)$. Conclusion: The findings of the study confirm the importance of searching actively the presence of mobility deficit in the elderly, particularly in Primary Health services.

Keywords: Elderly; accidental falls; mobility. 


\section{Introdução}

O progressivo aumento populacional do contingente de idosos traz à tona questôes inerentes a esta faixa etária, principalmente as que são relacionadas à capacidade funcional, ocorrência de doenças crônicas não transmissíveis e associação de graus variados de incapacidade a processos mórbidos crônicos ${ }^{1}$.

A perda da capacidade funcional é um dos principais problemas de saúde a atingir a população idosa, podendo acarretar diminuição das habilidades físicas necessárias para realização das atividades de vida diária (AVDs), as quais envolvem os cuidados pessoais, além de comprometer as atividades instrumentais de vida diária (AIVDs), que se associam a tarefas mais complexas do cotidiano.

Entre os eventos incapacitantes que acometem a população idosa e que podem causar ou estar associados à incapacidade funcional, destaca-se a ocorrência de queda, que consiste no tipo de lesão mais comum neste grupo ${ }^{2,3}$.

As quedas representam um evento bastante temido pelos idosos, especialmente pelas complicaçóes que a elas se associam. No Brasil, num estudo longitudinal realizado no município de São Paulo com idosos da comunidade, evidenciou-se que cerca de $31 \%$ deles referiam ter tido ao menos uma queda no ano precedente à pesquisa ${ }^{4}$.

Lebrão et al. ${ }^{5}(2005)$ constataram que $28,6 \%$ dos idosos avaliados no Projeto SABE relataram quedas, e a frequência destas aumentou de acordo com a idade, sendo registrada em $26,2 \%$ dos idosos entre 60 e 74 anos, com aumento para $36,9 \%$ em indivíduos a partir de 75 anos. Nesse estudo, a presença desse evento foi maior entre as mulheres do que entre os homens.

Cerca de 10 a $15 \%$ das quedas resultam em lesóes sérias, como fratura de colo de fêmur, traumatismo crânio encefálico, entre outras. Estima-se que esta seja a décima quinta causa de morte entre os idosos. Outras consequências importantes são: lesôes graves de partes moles, dor, restrição de mobilidade, medo de quedas recorrentes, depressão, isolamento social, hospitalização, risco de internação em instituições de longa permanência e morte ${ }^{7}$. Além disso, as quedas também contribuem para o surgimento de novas condiçôes mórbidas, desencadeando maior demanda dos serviços de saúde com aumento do número de consultas, atendimentos especializados, maior custo e tempo de internação ${ }^{8}$.

Considerando-se a relevância do evento "quedas", e a possibilidade de declínio funcional associada ao envelhecimento, buscou-se traçar o perfil de risco de idosos assistidos na Atenção Primária em um grande centro urbano, que representa o nível em que, preferencialmente, deve ser conduzida a maior parte dos atendimentos à população idosa. Confirmando a importância desse tipo de estudo, destaca-se, ainda, a constatação de que se carece da implantação e desenvolvimento efetivo de políticas de saúde que viabilizem medidas preventivas para esse tipo de agravo, o que supóe o reconhecimento dos fatores a ele associados.

\section{Métodos}

Desenvolveu-se um estudo de corte transversal realizado em dois centros de saúde: Jardim Paranapanema e Jardim Conceição, localizados respectivamente nos distritos sul e leste do município de Campinas (SP), ambos integrantes do Sistema Único de Saúde (SUS). A amostra foi composta por 70 idosos, de ambos os sexos, não institucionalizados, regularmente assistidos por equipe de profissionais dessas unidades citadas.

Os critérios de inclusão foram ter idade igual ou superior a 60 anos, deambular sem o uso de órtese; compreender as instruçóes apresentadas pelo pesquisador. Foram excluídos os idosos que apresentassem: enfermidade que impossibilitasse assumir a posição ortostática; cadeirantes; aqueles com incapacidade de compreensão para realizar as instruçóes do pesquisador.

Para estudar o grau de independência, procedeu-se à avaliaçãoo das Atividades de Vida Diária (AVDs), por meio da Medida da Independência Funcional (MIF), que permite estabelecer graus de independência quanto a qualquer tipo de auxílio, físico ou verbal. Esses dados foram colhidos diretamente com o paciente por uma ficha de avaliação, podendo haver auxílio do acompanhante ${ }^{9,10}$.

Para o presente estudo, considerando-se a MIF, foram utilizados os totais de pontos encontrados nos itens "autocuidado" (escore de 7 a 42), "controle de esfíncteres" (escore de 2 a 14) e para os valores totais dos itens "transferências" e "locomoção" que somados foram incluídos na categoria "mobilidade" (escore de 5 a 35).

Os idosos foram selecionados de forma aleatória pelo período de três meses, considerando-se os que eram atendidos em consultas previamente agendadas em um dos Centros de Saúde (CS) em que se deu a pesquisa. Os que preenchiam os critérios para participação no presente estudo e concordavam em participar deste foram encaminhados pelos profissionais desses dois CS ao pesquisador, que avaliou todos os casos no mesmo espaço físico desses serviços.

Antes de se submeterem à avaliação, todos os participantes foram previamente informados e esclarecidos sobre o projeto de pesquisa e seus objetivos. A seguir, assinaram o Termo de Consentimento Livre e Esclarecido para serem incluídos nesse estudo. $\mathrm{Na}$ impossibilidade de se obter a assinatura do paciente, esta foi obtida de acompanhante que se responsabilizou por ele. O protocolo de avaliação teve duração média de 30 minutos.

Para descrever o perfil da amostra segundo as variáveis em estudo, foram feitas tabelas de frequência das 
variáveis categóricas, com valores de frequência absoluta (n) e percentual (\%), e estatísticas descritivas das variáveis numéricas. Para comparação das principais variáveis categóricas foram utilizados os testes qui-quadrado ou exato de Fisher (para valores esperados menores que 5), e o teste de Mann-Whitney para comparação de variáveis numéricas entre os grupos com e sem relato de quedas. Para analisar a relação entre as variáveis numéricas, foi utilizado o coeficiente de correlação de Spearman, devido à ausência de distribuição normal das variáveis. O nível de significância adotado para os testes estatísticos foi de 5\%, utilizando-se o seguinte programa computacional: "The SAS System for Windows" (Statistical Analysis System), versão 8.02. SAS Institute Inc, 19992001, Cary, NC, USA.

Os idosos que apresentaram, durante a avaliação, algum tipo de alteração que evidenciasse maior risco para quedas, foram encaminhados ao serviço de fisioterapia dos respectivos centros de saúde.

Este trabalho foi previamente submetido ao Comitê de Ética em Pesquisa da Faculdade de Ciências Médicas da Universidade Estadual de Campinas, tendo sido aprovado conforme registro 598/2008.

\section{Resultados}

A amostra foi composta por 70 idosos, sendo que 52 eram mulheres $(74,29 \%)$. Apresentavam idade média de 70,96 ( $\pm 6,63)$ anos, sendo que 32 estavam na faixa entre 60 e 69 anos (45,71\%), 29 tinham idade entre 70 e 79 anos $(41,43 \%)$ e nove tinham 80 anos ou mais $(12,86 \%)$. Quanto à escolaridade, nove eram analfabetos $(12,86 \%), 43(61,43 \%)$ idosos tinham até quatro anos de escolaridade e $18(25,71 \%)$ tinham mais que quatro anos de educação formal. Em relação ao estado conjugal, $33(47,14 \%)$ eram casados, $28(40 \%)$ eram viúvos, seis $(8,57 \%)$ eram divorciados e três $(4,29 \%)$ solteiros. 41 idosos relataram quedas $(58,57 \%)$ e 29 $(41,43 \%)$ negaram esse tipo de ocorrência, com uma média de $1,07( \pm 1,61)$ queda no ano anterior à avaliação. Entre os que relataram quedas, $39(95,12 \%)$ tiveram entre uma a cinco quedas, e apenas dois $(4,88 \%)$ tiveram seis ou mais quedas.

Em relação aos achados da MIF (tabela 1), obtiveram pontuação máxima no domínio autocuidado: 63 (90\%) idosos no item alimentação, 60 (85,71\%) no item higiene

Tabela 1: Pontuação dos domínios da MIF em 70 idosos assistidos em dois centros de saúde do município de Campinas (SP)

\begin{tabular}{|c|c|c|c|c|c|c|c|c|}
\hline \multicolumn{9}{|c|}{ MIF - AUTOCUIDADO } \\
\hline Alimentaçáo & n & $\%$ & Higiene Pessoal & $\mathbf{n}$ & $\%$ & Banho & $\mathbf{n}$ & $\%$ \\
\hline$\leq 5$ & 03 & 4,29 & $\leq 5$ & 02 & 2,86 & $\leq 5$ & 04 & 5,72 \\
\hline 6 & 04 & 5,71 & 6 & 08 & 11,43 & 6 & 11 & 15,71 \\
\hline 7 & 63 & 90,0 & 7 & 60 & 85,71 & 7 & 55 & 78,57 \\
\hline $\begin{array}{c}\text { Vestir-se da cintura } \\
\text { para cima }\end{array}$ & n & $\%$ & $\begin{array}{c}\text { Vestir-se da cintura } \\
\text { para baixo }\end{array}$ & $\mathbf{n}$ & $\%$ & $\begin{array}{c}\text { Uso do vaso } \\
\text { sanitário }\end{array}$ & $\mathbf{n}$ & $\%$ \\
\hline$\leq 5$ & 03 & 4,29 & $\leq 5$ & 04 & 5,71 & $\leq 5$ & 01 & 1,43 \\
\hline 6 & 19 & 27,14 & 6 & 21 & 30,00 & 6 & 05 & 7,14 \\
\hline 7 & 48 & 68,57 & 7 & 45 & 64,29 & 7 & 64 & 91,43 \\
\hline \multicolumn{9}{|c|}{ MIF - CONTROLE DE ESFÍNCTERES } \\
\hline Controle de urina & $\mathbf{n}$ & $\%$ & Controle de fezes & $\mathbf{n}$ & $\%$ & & & \\
\hline$\leq 5$ & 12 & 17,13 & $\leq 5$ & 04 & 5,71 & & & \\
\hline 6 & 17 & 24,29 & 6 & 01 & 1,43 & & & \\
\hline 7 & 41 & 58,57 & 7 & 65 & 92,86 & & & \\
\hline \multicolumn{9}{|c|}{ MIF - MOBILIDADE } \\
\hline $\begin{array}{l}\text { Transferência do } \\
\text { leito para cadeira }\end{array}$ & $\mathbf{n}$ & $\%$ & $\begin{array}{c}\text { Transferência do } \\
\text { vaso sanitário }\end{array}$ & $\mathbf{n}$ & $\%$ & $\begin{array}{c}\text { Transferência } \\
\text { do banheiro } \\
\text { para } \\
\text { Chuveiro }\end{array}$ & $\mathbf{n}$ & $\%$ \\
\hline$\leq 5$ & 02 & 2,86 & $\leq 5$ & 01 & 1,43 & $\leq 5$ & 01 & 1,43 \\
\hline 6 & 29 & 41,46 & 6 & 12 & 17,14 & 6 & 04 & 5,71 \\
\hline 7 & 39 & 55,71 & 7 & 57 & 81,43 & 7 & 65 & 92,86 \\
\hline Marchas & n & $\%$ & Escadas & $\mathrm{n}$ & $\%$ & & & \\
\hline$\leq 5$ & 0 & 0 & $\leq 5$ & 03 & 4,29 & & & \\
\hline 6 & 07 & 10,0 & 6 & 48 & 68,57 & & & \\
\hline 7 & 63 & 90,0 & 7 & 19 & 27,14 & & & \\
\hline
\end{tabular}


pessoal, 55 (78,57\%) em banho, 48 (68,57\%) em vestir-se da cintura para cima, $45(64,29 \%)$ em vestir-se da cintura para baixo e $64(91,43 \%)$ no item uso do vaso sanitário. No domínio controle de esfíncteres, 41 (58,57\%) idosos atingiram pontuação máxima no item controle de urina e $65(92,86 \%)$ no item controle de fezes. Finalmente, no domínio mobilidade, a pontuação máxima foi atingida por $39(55,71 \%)$ idosos no item transferência do leito para cadeira, $57(81,43 \%)$ na transferência do vaso sanitário, $65(92,86 \%)$ na transferência de banheiro e chuveiro, 63 (90\%) em marcha e $19(27,14 \%)$ em subir escadas. $\mathrm{Na}$ MIF autocuidado, a pontuação média foi de 40,4 ( \pm 3,03); para a MIF controle de esfíncteres, a pontuação média foi de 13,20 ( $\pm 1,27)$ e, para a MIF mobilidade, a média foi de 33,36 $( \pm 1,53)$.

$\mathrm{Na}$ tabela 2, registraram-se as variáveis que apresentavam ou não associação com o autorrelato de quedas no ano anterior à pesquisa. Apenas gênero $(\mathrm{p}=0,049)$, pontuação da MIF no domínio autocuidado ( $\mathrm{p}=$ $0,019)$ e no domínio mobilidade ( $\mathrm{p}=0,040)$ evidenciaram associação com o relato de quedas. Efetivamente, constatou-se maior número de mulheres idosas entre os que referiram quedas, com 82,93\% (34) de respostas positivas em comparação a 17,07\% (07) dos homens idosos. No grupo que náo relatava quedas, 18 (62,07\%) eram mulheres e $11(37,93 \%)$ eram homens.

Tabela 2: Comparação das principais variáveis categóricas em estudo quanto à ocorrência de queda em 70 idosos assistidos em dois centros de saúde do município de Campinas (SP)

\begin{tabular}{|c|c|c|c|}
\hline & $\begin{array}{c}\text { Quedas } \\
+ \\
\end{array}$ & Quedas & $\mathbf{p}$ \\
\hline Idade (anos) & 71,93 & 69,59 & 0,116 \\
\hline Gênero & & & 0,049 \\
\hline Feminino & $34(82,93 \%)$ & $18(62,07 \%)$ & \\
\hline Masculino & $07(17,07 \%)$ & $11(37,93 \%)$ & \\
\hline Procedência & & & 0,417 \\
\hline Rural & $16(39,02 \%)$ & $11(37,93 \%)$ & \\
\hline Urbana & $25(60,98 \%)$ & $18(62,07 \%)$ & \\
\hline Situação conjugal & & & 0,926 \\
\hline Com companheiro & $21(51,22 \%)$ & $12(41,38 \%)$ & \\
\hline Sem companheiro & $20(48,78 \%)$ & $17(58,62 \%)$ & \\
\hline Escolaridade formal & & & 0,921 \\
\hline Analfabeto & $05(12,20 \%)$ & $04(13,79 \%)$ & \\
\hline 1 a 4 anos & $26(63,41 \%)$ & $17(58,62 \%)$ & \\
\hline$>4$ anos & $10(24,35 \%)$ & $08(27,59 \%)$ & \\
\hline $\begin{array}{l}\text { MIF autocuidado } \\
\text { (pontuação total) }\end{array}$ & $39,80 \pm 3,70$ & $41,24 \pm 1,38$ & 0,019 \\
\hline $\begin{array}{c}\text { MIF controle de } \\
\text { esfíncteres (pontuação } \\
\text { total) }\end{array}$ & $13,07 \pm 1,42$ & $13,38 \pm 1,01$ & 0,254 \\
\hline $\begin{array}{l}\text { MIF mobilidade } \\
\text { (pontuação total) }\end{array}$ & $33,05 \pm 1,67$ & $33,79 \pm 1,21$ & 0,040 \\
\hline
\end{tabular}

Conforme se pode verificar na tabela 3, o presente estudo evidenciou correlaçáo significativa entre relato de quedas e pontuação da MIF no domínio mobilidade $(p=0,0302)$. Também se registrou correlação significativa entre a idade e a pontuação da MIF nos domínios controle de esfíncteres $(\mathrm{p}=0,0111)$ e mobilidade $(\mathrm{p}=$ 0,0067).

Tabela 3: Correlação entre quedas, idade e pontuação total nos domínios da MIF

\begin{tabular}{ccccc}
\hline & & $\begin{array}{c}\text { MIF- } \\
\text { Autocuidado }\end{array}$ & $\begin{array}{c}\text { MIF - } \\
\text { Controle de } \\
\text { Esfíncteres }\end{array}$ & $\begin{array}{c}\text { MIF - } \\
\text { Mobilidade }\end{array}$ \\
\hline Idade & $\mathrm{r}$ & $-0,20788$ & $-0,30186$ & $-0,32140$ \\
& $\mathrm{p}$ & 0,0842 & 0,0111 & 0,0067 \\
$\begin{array}{c}\text { Relato } \\
\text { de quedas }\end{array}$ & $\mathrm{r}$ & $-0,21243$ & $-0,23144$ & $-0,25925$ \\
& $\mathrm{p}$ & 0,0775 & 0,0539 & 0,0302 \\
\hline
\end{tabular}

\section{Discussão}

É importante destacar que neste estudo se registrou maior proporção de mulheres idosas, à semelhança do que se verifica em registros feitos pelo Instituto Brasileiro de Geografia e Estatística (IBGE), em relação à população idosa brasileira. $\mathrm{Na}$ Pesquisa Nacional por Amostra de Domicílio (PNAD) ${ }^{13}$, divulgada em 2011 pelo IBGE, para a população a partir de 60 anos de idade, foram registrados 13,111 (55,71\%) milhôes de mulheres e 10,423 (44,29\%) milhóes de homens. Esse achado pode ser relacionado, entre outros fatores, ao maior cuidado das mulheres com a saúde e aos programas de saúde da mulher existentes na Rede Pública de Saúde, que possibilitam a prevenção e o diagnóstico precoce de enfermidades crônicas e das complicaçóes a elas associadas. De qualquer modo, a literatura evidencia uma maior sobrevida de mulheres em relaçáo aos homens, o que resulta em aumento da proporção de idosas em relação aos idosos, fenômeno este conhecido como "feminização da velhice" ${ }^{14}$.

Neste estudo, as mulheres tiveram maior relato de quedas $(82,93 \%)$, o que também está em consonância com os dados da literatura ${ }^{15,16}$. Em estudo brasileiro de Gawryszewisk et al. ${ }^{17}$, ao se avaliar as internaçôes por causas externas em indivíduos com 60 anos e mais, no ano 2000, das 48.940 hospitalizaçóes por quedas, as mulheres respondiam por 29.491 , ou seja, $60,26 \%$ do total. Entretanto, os fatores associados a esse fenômeno ainda precisam de melhor esclarecimento, sugerindo-se como possíveis causas: maior fragilidade das mulheres em relação aos homens, assim como a maior prevalência de doenças crônicas, ou ainda, a maior exposição às atividades domésticas ${ }^{4}$. 
Também se evidenciou maior proporção de idosos na faixa de 60 a 69 anos (45,71\%), seguidos por aqueles com idade entre 70 e 79 anos $(41,43 \%)$ e $\geq 80$ anos $(12,86 \%)$, dados esses que se assemelham aos encontrados na PNAD $(2011)^{13}$, que mostrou que $55,2 \%$ da população idosa se encontravam na faixa etária entre 60 a 69 anos; $30,5 \%$ entre 70 a 79 anos e $13,2 \%$ na faixa etária de 80 anos ou mais.

Considerando-se, ainda, a idade, na amostra avaliada não se registrou diferença entre os idosos que relataram e os que não relataram quedas, embora para o primeiro grupo esse fosse um pouco maior. Esse achado se contrapóe à literatura que registra associação entre aumento da idade e maior ocorrência de quedas, o que pode ser associado à instalação progressiva dos declínios funcionais e maior ocorrência de doenças crônicas em idade mais avançada ${ }^{18}$. Nesta pesquisa, também se constatou que os mais idosos tinham pior desempenho nos domínios controle de esfíncteres e mobilidade da MIF. Dessa forma, uma possibilidade que precisa ser considerada é a de que os mais idosos tenham deixado de relatar as quedas, seja por esquecimento, por medo de sofrerem restriçóes às suas atividades, ou por outras causas. Esse fato pode ter acarretado uma menor estimativa desse evento entre os idosos nas faixas etárias mais avançadas.

Nesta amostra, predominaram os idosos com até quatro anos de escolaridade $(61,43 \%)$, registrando-se analfabetismo em $12,86 \%$ da amostra, o que também está em consonância com os achados do $\mathrm{IBGE}^{19}$, que também confirmou que, no Brasil, o analfabetismo está concentrado nas faixas etárias mais elevadas. Entretanto, o estudo náo evidenciou associação entre o nível de escolaridade e o autorrelato de quedas.

Constatou-se ainda que $41(58,57 \%)$ idosos da amostra relatavam ocorrência de quedas no ano precedente, o que representou, em média, 1,07 queda por ano. Esses dados evidenciam um maior relato de quedas na presente amostra, quando comparada aos achados de Perracini e Ramos ${ }^{4}$, em estudo populacional desenvolvido no município de São Paulo, nos anos de 1991/92 e 1994/95, em que se encontrou autorrelato de quedas em cerca de $31 \%$ dos idosos avaliados. Pode-se supor que, no estudo atual, tenha ocorrido um viés de encaminhamento por parte dos profissionais dos centros de saúde no sentido de selecionarem idosos que haviam relatado quedas, registrando-se, dessa forma, um predomínio daqueles com autorrelato positivo. Outra possibilidade a considerar é a de que os integrantes do presente trabalho foram selecionados a partir de uma população idosa assistida em serviços de Atenção Primária, sendo aí maior o registro de declínio funcional e de enfermidades crônicas.

Os idosos com relato de quedas apresentaram diferença significativa dos que negavam quedas, quando avaliados por meio da MIF, o que se evidenciou pela menor pontuação nessa escala, justamente nos domínios autocuidado e mobilidade. Verificou-se associação entre relato de quedas e pior avaliação na MIF mobilidade, o que está em consonância com as pesquisas que mostram que idosos com déficit nas atividades de vida diária (AVD) apresentam maior chance de sofrer quedas $^{25}$. Segundo o que foi verificado por Perracini e Ramos ${ }^{9}$, idosos com comprometimento funcional apresentavam 1,71 vez mais chance de sofrer queda. A restrição nas AVD em decorrência de queda pode estar relacionada a algum prejuízo físico e/ou ao medo de sofrer nova queda ${ }^{25}$.

Nesta pesquisa, não se encontrou associação entre autorrelato de quedas e pior desempenho no domínio controle de esfíncteres, embora se tenha observado uma tendência à associação $(\mathrm{p}=0,0539)$. Esse dado diverge de achados da literatura, que mostram que a incontinência urinária se vincula a um maior relato de quedas, muito provavelmente em decorrência da urgência do idoso em chegar a tempo ao banheiro que se soma a uma menor mobilidade ${ }^{26,27}$.

Avaliando o domínio controle de esfíncteres da MIF, constatou-se correlação negativa com a idade. Sabese que a perda do controle esfincteriano pode ocorrer nos idosos, em parte, por obstrução da uretra, o que se deve, provavelmente, à complacência uretral diminuída ou ausente; esta também pode estar associada à falta de contratilidade do músculo detrusor. Tais alteraçóes tendem a se acentuar com o envelhecimento ${ }^{34}$.

Em relação à mobilidade avaliada pela MIF, também se obteve correlação negativa com a idade. Mais uma vez achados da literatura confirmam esse resultado, conforme demonstrado por Ricci ${ }^{20}$, que encontrou comprometimento no item "escadas" da MIF, que está incluído no domínio mobilidade. Entretanto, Paula ${ }^{35}$ não evidenciou diferença significativa nem correlação entre faixa etária e pontuação da MIF.

\section{Conclusão}

No presente trabalho, o autorrelato de quedas correlacionou-se positivamente com menor pontuação no domínio "mobilidade" da MIF ( $\mathrm{p}=0,0302)$. Além disso, o registro de déficit nesse domínio e no controle de esfíncteres mostrou correlação com idade mais avançada. Esses achados estão em consonância com a literatura que evidencia que o avanço da idade e a perda da independência funcional aumentam a carga de morbidade junto à população idosa, inclusive no que se refere ao evento queda e suas complicaçóes.

Seguramente, mais pesquisas se fazem necessárias para melhor caracterizar a associação entre os diferentes 
graus de perda da capacidade funcional e a ocorrência de queda, porém, embora este seja um estudo transversal com uma amostra pequena de idosos, pode-se destacar que o ele permite confirmar a relevância de buscar ativamente a presença de déficit de mobilidade em idosos, particularmente nos serviços de Atenção Primária.

\section{Referências}

1. Ricci NA, Kubota MT, Cordeiro RC. Concordância de observaçóes sobre a capacidade funcional de idosos em assistência domiciliar. Rev Saúde Publica. 2005; 39(4): 655-62.

2. Santos LD, Salmela LFT, Lelis FO. Eficácia da Atividade Física na manutenção do desempenho funcional do idoso: revisão de literatura. Rev Fisioter Bras. 2001; 2 (3): 169-77.

3. Foster A, Young J. Incidence and consequences of falls due to stroke: a systematic inquiry. British Med J. 1995; 311 : 83-6.

4. Perracini MR, Ramos LR. Fatores associados a quedas em uma coorte de idosos residentes na comunidade. Rev Saúde Pública. 2002; 36(6): 709-16.

5. Lebrão ML, Laurenti R. Saúde, bem-estar e envelhecimento: o estudo SABE no município de São Paulo. Rev Bras de Epidemiol. 2005; 8(2): 127-41.

6. McIlroy WE, Maki BE. Age-related changes in compensatory stepping in response to unpredictable perturbations. J. Gerontol. 1996; 51: 289-96.

7. Moilan KC, Binder EF. Falls in older adults: Risk assessment, management and prevention. Am J Med. 2007; 120: 493-97.

8. Lebrão ML, Duarte YAO. O Projeto SABE no município de São Paulo: uma abordagem inicial. 1 ed. São Paulo: Athalaia Bureau; 2003.

9. Granger CV, Hamilton BB, Keith RA, Zielezny M, Sherwin FS. Advances in functional assessment for rehabilitation. IN: Lewis CB, editor. Topics in Geriatric Rhabilitation. Baltimore: Aspen; 1986: 59-74.

10. Riberto M, Miyazaki MH, Jucá SSH, Sakamoto H, Pinto PPN, Battistella LR. Validação da versáo brasileira da medida de independência funcional. Acta Fisiatr. 2004; 119 (2): 72-6.

11. Shumway-Cook A, Horak FB. Assessing the influence of sensory interaction on balance: suggestion from the field. Phys Ther. 1986; 66: 1548-50.

12. Kendall FP, McCreary EK, Provance PG. Músculos: provas e funçôes. 4 ed. São Paulo: Manole; 1995.

13. PNAD. Pesquisa Nacional Por Amostra de Domicílio. IBGE. [citado em: 02 mai. 2013]. Disponível em: http:// www.ibge.gov.br/home/presidencia/noticias/imprensa/ppts /00000010135709212012572220530659

14. Neri AL. Feminização da velhice. IN: Neri AL, organizadora. Idosos no Brasil. Vivências, Desafios e Expectativas na Terceira Idade. São Paulo: Ediçóes SESCSP \& Editora Fundação Perseu Abramo; 2007: 47-64.
15. Chu LW, Chi I, Chiu AYY. Incidence and predictors of falls in the Chinese elderly. Ann Acad Med Singapore. 2005; 34: 60-72.

16. Píton DA. Análise dos fatores de risco de quedas em idosos: estudo exploratório em instituição de longa permanência no município de Campinas [dissertação]. Campinas (SP): Universidade Estadual de Campinas; 2004.

17. Gawryszewski VP, Jorge MHPM, Koizumi MS. Mortes e internaçóes por causas externas entre os idosos no Brasil: desafio de integrar a saúde coletiva e atenção individual. Rev Assoc Med Bras. 2004; 50(1): 97-103

18. Russel MA, Hill KD, Blackberry I, Day LL, Dharmage SC. Falls risk and functional decline in older fallers discharged directly from emergency departments. J. Geront. 2006; 61a (10): 1090-95.

19. IBGE. Instituto Brasileiro de Geografia e Estatística. [citado em: 14 mai. 2013]. Disponível em: ftp://ftp.ibge.gov.br/ Trabalho_e_Rendimento/Educacao_e_Trabalho

20. Cohen H, Blatchly CA, Gombash, LL. A study of the clinical test of sensory interaction and balance. Phys Ther. 1993; 73 (6): 346-51.

21. Ricci NA. Influência das informaçóes sensoriais no equilíbrio estático de idosos da comunidade: comparação em relação ao histórico de quedas [dissertação]. Campinas (SP): Universidade Estadual de Campinas; 2006.

22. Sabchuk RAC, Bento PCB, Rodacki ALF. Comparação entre testes de equilíbrio de campo e plataforma de força. Rev Bras Med Esporte. 2012; 18(6): 404-8.

23. Gonçalves DFF, Ricci NA, Coimbra AMV. Equilíbrio funcional de idosos da comunidade: comparação em relação ao histórico de quedas. Rev Bras Fisioter. 2009; 13(4): 316-23.

24. Gai J, Gomes L, Nóbrega OT, Rodrigues MP. Fatores associados a quedas em mulheres idosas residentes na comunidade. Rev Assoc Med Bras. 2010; 56(3): 327-32.

25. Gonança FF, Gazzola JM, Aratani MC, Perracini MR, Gonança MM. Circunstâncias e consequências de quedas em idosos com vestibulopatia crônica. Rev Bras Otorrinolaringol. 2006; 72(3): 388-93.

26. Rekeneire N, Visser M, Peila R, Nevitt MC,Cauley JA, Tylavsky FA et al. Is a fall just a fall: correlates of falling in healthy older persons. The health, aging and body composition study. J Am Ger Soc. 2003; 51(6): 841-6.

27. Kron M, Loy S, Sturm E, Nikolaus T, Becker C.Risk indicators for falls in institutionalized frail elderly. Am J Epidemiol. 2003; 158(7): 645-53.

28. Dean JC, Kuo AD, Alexander NB. Age - related changes in maximal hip strength and movement speed. J Geron. 2004; 59a (3): 286-92.

29. Ishizuka MA. Avaliação e comparação dos fatores intrínsecos dos riscos de quedas em idosos com diferentes estados funcionais. [dissertação]. Campinas (SP): Universidade Estadual de Campinas; 2003.

30. Queiroz L, Lirac S, Sasaki A. Identificação do risco de quedas pela avaliaçáo da mobilidade funcional em idosos 
hospitalizados. Rev Baiana de Saúde Pública. 2009; 33(4): 534-43.

31. Alencar MA, Arantes PMM, Dias JMD, Kirkwood RN, Pereira LSM, Dias RC. Muscular function and functional mobility of fallers and non-fallers elderly women with osteoarthritis of the knee. Braz J Med Biol Res. 2007; 40(2): 277-83.

32. Kuh D, Bassey EJ, Butterworth S, Hardy R, Wadsworth MEJ. Grip strength, postural control, and functional leg power in a representative cohort of British men and women: associations with physical activity, health status, and socioeconomic conditions. J Geront. 2005; 60(2): 224-31.

33. Foldvari M, Clark M, Laviolette LC, Bernstein MA, Kaliton D, Castaneda C, Pu CT, Hausdorff JM, Fielding RA, Singh
MA. Association of muscle power with functional status in community-dwelling elderly women. J Gerontol. 2000; 55(4): 192-9.

34. McGuire EJ, Fitzpatrick CC, Wan J, Bloom D, Sanvordenker J, Ritchey M, Gormley EA. Clinical assessment of urethral sphincter function. J Urol. 1993; 150: 1452-4.

35. Paula JAM. Avaliação do idoso: capacidade funcional, independência e sua relação com outros indicadores de saúde [tese]. Campinas (SP): Universidade Estadual de Campinas; 2007.

36. Roubenoff R. Sarcopenia: Effects on body composition and function. J Geront, 2003; 58(11): 1012-7. 